\title{
Application of Long-Range Surface Plasmon Resonance for ABO Blood Typing
}

\author{
Wanida Tangkawsakul, ${ }^{1,2}$ Toemsak Srikhirin, ${ }^{2}$ Kazunari Shinbo, ${ }^{1}$ \\ Keizo Kato, ${ }^{1}$ Futao Kaneko, ${ }^{1}$ and Akira Baba ${ }^{1}$ \\ ${ }^{1}$ Graduate School of Science and Technology and Center for Transdisciplinary Research, Niigata University, \\ 8050 Ikarashi 2-Nocho, Nishi-ku, Niigata 950-2181, Japan \\ ${ }^{2}$ Materials Science and Engineering Programme, Multidisciplinary Unit and Center of Intelligence Materials and Systems, \\ NANOTEC Center of Excellence at Mahidol University, Department of Physics, Faculty of Science, Mahidol University, \\ Rama 6 Rd., Phayathai, Rajathavee, Bangkok 10400, Thailand
}

Correspondence should be addressed to Toemsak Srikhirin; sctsk@mahidol.ac.th and Akira Baba; ababa@eng.niigata-u.ac.jp

Received 12 September 2016; Accepted 30 November 2016

Academic Editor: Günther K. Bonn

Copyright ( 2016 Wanida Tangkawsakul et al. This is an open access article distributed under the Creative Commons Attribution License, which permits unrestricted use, distribution, and reproduction in any medium, provided the original work is properly cited.

In this study, we demonstrate a long-range surface plasmon resonance (LR-SPR) biosensor for the detection of whole cell by captured antigens A and B on the surface of red blood cells (RBCs) as a model. The LR-SPR sensor chip consists of high-refractive index glass, a Cytop film layer, and a thin gold $(\mathrm{Au})$ film, which makes the evanescent field intensity and the penetration depth longer than conventional SPR. Therefore, the LR-SPR biosensor has improved capability for detecting large analytes, such as RBCs. The antibodies specific to blood group A and group B (Anti-A and Anti-B) are covalently immobilized on a grafting self-assembled monolayer (SAM)/Au surface on the biosensor. For blood typing, RBC samples can be detected by the LR-SPR biosensor through a change in the refractive index. We determined that the results of blood typing using the LR-SPR biosensor are consistent with the results obtained from the agglutination test. We obtained the lowest detection limits of $1.58 \times 10^{5} \mathrm{cells} / \mathrm{ml}$ for RBC-A and $3.83 \times$ $10^{5}$ cells $/ \mathrm{ml}$ for RBC-B, indicating that the LR-SPR chip has a higher sensitivity than conventional SPR biosensors $\left(3.3 \times 10^{8} \mathrm{cells} / \mathrm{ml}\right)$. The surface of the biosensor can be efficiently regenerated using $20 \mathrm{mM} \mathrm{NaOH}$. In summary, as the LR-SPR technique is sensitive and has a simple experimental setup, it can easily be applied for $\mathrm{ABO}$ blood group typing.

\section{Introduction}

Blood group typing is necessary in the treatment of patients with massive blood loss. Matching the donor's blood group with the patient's blood group is required before transfusion to avoid a blood incompatibility event. The ABO blood typing system is tested first for all blood transfusions, because it can cause serious damage in all other blood systems due to its strong specific antigen-antibody interactions. The $\mathrm{ABO}$ blood group system is classified based on the inherited properties of red blood cells (RBCs). It is determined by the presence or absence of certain proteins and oligosaccharides called antigens, including $\mathrm{A}$ and $\mathrm{B}$, which are presented on the surface of RBCs. A, B, and O RBCs structures are similar, but the difference is that A-type RBCs have
$\mathrm{N}$-acetylgalactosamine (GalNac) and B-type RBCs have galactose (Gal), while O-type RBCs have neither. The blood group is associated with Anti-A and Anti-B immunoglobulin $\mathrm{M}$ antibodies (IgM), which are the body's natural defense against foreign antigens. The $\mathrm{ABO}$ blood typing system breaks blood types down into four groups: $\mathrm{A}, \mathrm{B}, \mathrm{AB}$, or $\mathrm{O}$. Each group specifies the antigens and antibodies found in that individual, such as the $\mathrm{A}$ antigens and $\mathrm{B}$ antibodies found in blood group $\mathrm{A}$, the $\mathrm{B}$ antigens and $\mathrm{A}$ antibodies found in blood group $B$, both $A$ and $B$ antigens found in blood group $\mathrm{AB}$, and both $\mathrm{A}$ and $\mathrm{B}$ antibodies found in blood group $\mathrm{O}[1-$ 5].

Surface plasmon resonance (SPR) technique has become a widely used technique for the detection of biomolecular interactions since the 1990s. Among other applications, SPR 
sensor is often used in clinical diagnosis for the detection of antigen-antibody binding, protein-ligand interaction, and DNA detection [6-12]. SPR is a sensitive technique that can be applied for real time monitoring of the biomolecular interactions in solution $[13,14]$. The SPR biosensor is based on changes of the optical reflectivity by the adsorption of biomolecules on a gold $(\mathrm{Au})$ surface, which cause a change in refractive index near the SPR-active gold surface. Longrange surface plasmon resonance (LR-SPR) involves surface plasmons (SPs) that propagate along a thin metallic film embedded between two dielectric materials with similar refractive indices, for which both the evanescent field intensity and the penetration depth (more than $1 \mu \mathrm{m}$ ) are greater than those for conventional short-range SPs (penetration depth is $\sim 200 \mathrm{~nm}$ ) [15-20]. Hence, it is expected that the sensitivity at longer distances from the metal surface will increase with LR-SPR spectroscopy. Recently, Homola et al. reported that the LR-SPR biosensor could detect large analytes, such as the bacteria Escherichia coli $(0.7-1.0 \mu \mathrm{m})$ due to the large penetration depth of the evanescent wave [21, 22]. In addition, the LR-SPs enhanced the optical field wave at the metal-dielectric interface, leading to a higher sensor sensitivity and increased penetration into the analyte solution than that observed for conventional SPR. As a result, a thicker sensor coating with a significantly larger number of binding analyte molecules on the surface could be used [23, 24]. We have also previously reported an LR-SPR immunosensor based on the electrospun poly (acrylic acid) (PAA) fibers for the detection of human IgG [20].

The detection of antigens $A$ and $B$ on the surface of RBCs by conventional SPR was reported by Quinn et al. $[25,26]$. However, the detection of RBCs via conventional SPR is limited due to the penetration depth of evanescence field $(\sim 200 \mathrm{~nm})$, which is much less than the size of RBCs (i.e., $\sim 2 \mu \mathrm{m}$ thick and $\sim 7.5 \mu \mathrm{m}$ in diameter). Recently, various studies have been reported in the applications of SPR technique for the study of RBCs. Houngkamhang et al. [27] reported $\mathrm{ABO}$ blood typing via SPR imaging which observed interaction between immobilized Anti-A and Anti$\mathrm{B}$ antibodies array and $\mathrm{A}$ and $\mathrm{B}$ antigens on RBCs surface. The interaction of antigens around lower RBCs membrane surface and immobilized Anti-A and Anti-B antibodies array was applied to obtain the agglutination strength of the RBCs and immobilized antibody [28]. The SPR imaging, which utilized the shear force generated within the flow cell, was used to measure the rolling speed of red blood cell [28]. The technique can only extract the information at the interface because the limit of the evanescence field makes it impossible to study in whole cell. A number of important pieces of information regarding the property of the cell were undetectable such as cell elasticity and cell deformation. Krupin et al. used long-range surface plasmon waveguides for capturing only blood group antigen $\mathrm{A}$ on RBCs by immobilized Anti-A IgG via no comparative studies with Anti-B [29]. To our knowledge, there was no report regarding the detection of RBC by LR-SPR. Because the difference in surface chemistry, experimental set-up, and nature of SPR signal between LR-SPR and SPR, in this work, it is desirable to explore the possibility of applying LR-SPR to detect $\mathrm{ABO}$ blood group and understand the nature of the LR-SPR signal. Anti-A and Anti-B will be covalently immobilized on self-assembled monolayer (SAM) surface and $\mathrm{A}, \mathrm{B}, \mathrm{AB}$, and $\mathrm{O}$ will be detected by using LR-SPR. The Cytop ( $n=1.34$ ) fluoropolymer is used in the LRSPR system in order to match the refractive index with the phosphate buffered saline (PBS) buffer. All the results of blood typing were consistent with the results obtained from the agglutination test. The lowest detection amounts of RBC-A and RBC-B were $1.58 \times 10^{5}$ and $3.83 \times 10^{5}$ cells $/ \mathrm{ml}$ indicating that LR-SPR has much higher sensitivity than that obtained with conventional SPR biosensors [25, 26] and LRSPR waveguides [29]. We demonstrate that LR-SPR is a good candidate for classification of blood typing and has a high potential in other clinical applications such as bacteria cells, cancer, and rare RBCs.

\section{Experimental}

2.1. Chemicals and Materials. The 11-mercaptoundecanoic acid (11-MUA), phosphate buffered saline (PBS) tablets, and sodium acetate buffer ( $\mathrm{pH} 5$ ) were all purchased from SigmaAldrich. The 1-ethyl-3-(3-dimethylaminopropyl)-carbodiimide hydrochloride (EDC), N-hydroxysuccinimide (NHS), and ethanolamine hydrochloride (EA-HCl) were purchased from Tokyo Chemical Industry (TCI). The CTL-809M and CTL-180 solvents for the Cytop solution were purchased from Asahi glass. Mixed clones of monoclonal Anti-A and Anti-B and standard A cells, B cells, and $\mathrm{O}$ cells were obtained from the research unit of the Thai Red Cross Society.

\subsection{LR-SPR Instruments and LR-SPR Sensor Chip Fabrication.} The Kretschmann configuration is used for exciting surface plasmons using He-Ne laser with wavelength $(\lambda)$ of $632.8 \mathrm{~nm}$ $[30,31]$. LR-SPR was arranged to propagate along a thin metallic film embedded between two dielectrics with similar refractive index (Figure 1(a)). For the fabrication of the LRSPR sensor chip, a 7\% Cytop solution (dissolved 9\% Cytop (CTL-809M) in CTL-180 solvent) was spin-coated on a highrefractive index glass (OHARA S-LAH60, $n=1.83$ ) at a first spin rate of $500 \mathrm{rpm}$ for $10 \mathrm{~s}$ and second spin rate of $1300 \mathrm{rpm}$ for $20 \mathrm{~s}$. The Cytop solvent was dried at $180^{\circ} \mathrm{C}$ for $1 \mathrm{~h}$ in an oven, and the Cytop film (ca. $800 \mathrm{~nm}$ ) with a refractive index of 1.34 (similar to a refractive index of water, 1.33) was obtained on a high-refractive index glass. Then, $30 \mathrm{~nm}$ of gold $(\mathrm{Au})$ film was deposited on the Cytop film by vacuum evaporation. A chromium layer $(1 \mathrm{~nm})$ was used to promote Au adhesion with the Cytop film. As shown in Figure 1(b), the angular reflectivity curves of the conventional SPR and LRSPR chips measured in a bare Au/PBS buffer system indicate that the curve of the LR-SPR chip is sharper than that of the conventional SPR chip.

2.3. Immobilization of Antibodies and Detection of RBCs. The LR-SPR chip Au surface was covered by a self-assembled monolayer (SAM) using $10 \mathrm{mM}$ of 11-mercapto-undecanoic acid (11-MUA) in ethanol. The carboxylic groups of the SAM 


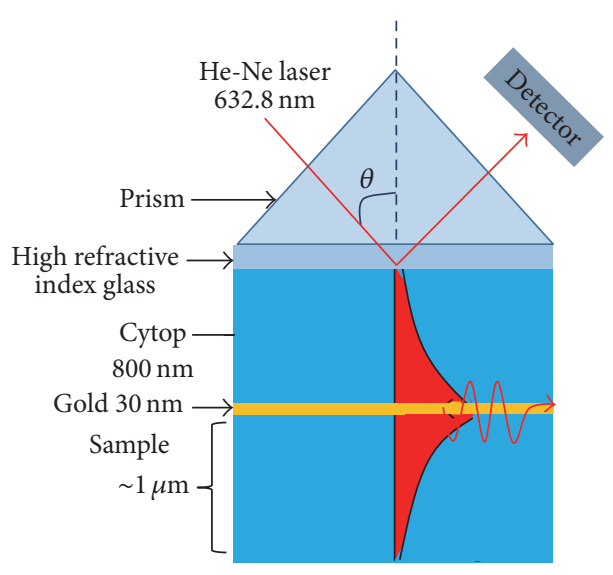

(a)

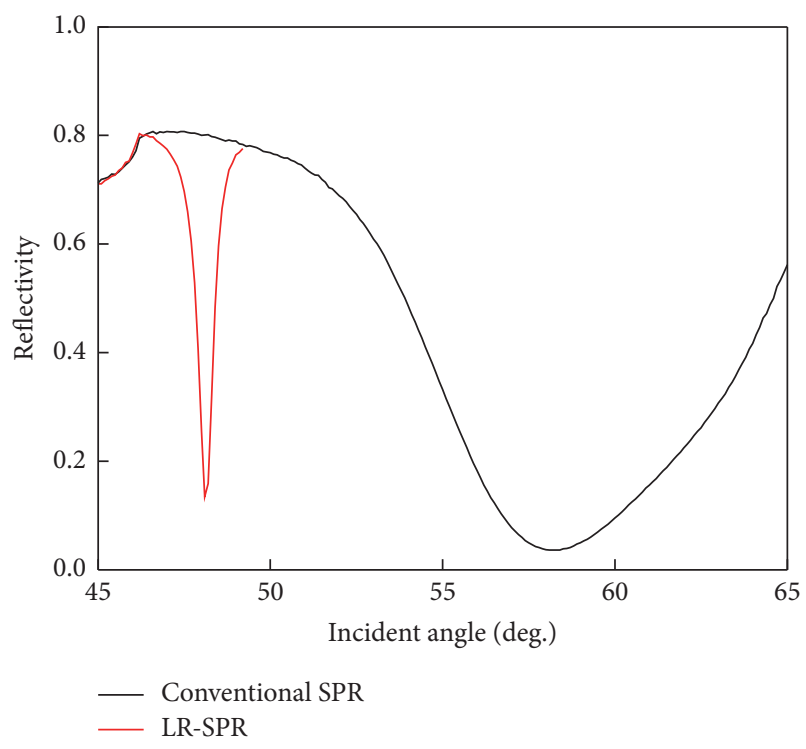

(b)

FIGURE 1: (a) Schematic of the long-range surface plasmon resonance (LR-SPR) setup. (b) The angular reflectivity curves of conventional SPR (prism-glass slide/gold (50 nm)/PBS buffer) and LR-SPR (prism-glass slide/Cytop ( $800 \mathrm{~nm}$ )/gold (30 nm)/PBS buffer).

surface were activated to their ester forms by immersion in 0.4 M EDC and 0.1 M NHS dissolved in deionized water at a ratio of $1: 1$. The monoclonal antibody for blood group A (Anti-A) and monoclonal antibody for blood group B (Anti-B) were covalently immobilized on the activated sensor surface (after rinsing the sensor chip with PBS buffer) by injecting the antibodies in sodium acetate at pH 5 at a $1: 10$ dilution onto the activated SAM surface [27]. The residual activated surface sites, which did not react with antibodies, were inactivated or blocked with $0.2 \mathrm{M}$ ethanolamine. The RBC samples from standard A, B, and $\mathrm{O}$ cells were detected on the immobilized antibody by observing the change in the refractive index on the LR-SPR biosensor. Standard RBC-A and RBC-B samples were diluted in the range of $3 \times 10^{4}$ to 3.8 $\times 10^{7}$ cells $/ \mathrm{ml}$. The number of cell number was counted using the hematocrit test. Regeneration of the LR-SPR chip surface was performed using $20 \mathrm{mM} \mathrm{NaOH}$, followed by rinsing with PBS running buffer. The LR-SPR measurements were carried out in a nonflow condition. A summary of the antibody immobilization on the biosensor chip and the detection of RBCs is shown in Figure 2.

\section{Results and Discussion}

3.1. Immobilization of Antibody and Detection of RBC-A. We compared the sensing ability between conventional SPR and LR-SPR for the detection and classification of RBC typing on grafted SAMs (11-MUA). First, the conventional SPR sensor chip was used for the evaluation of the immobilization of Anti-A/Anti-B on the sensor chip and the detection of RBCA. Angular reflectivity curves of the conventional SPR were observed before immobilization or after grafting the SAM, after immobilization of Anti-A, and after the detection of
RBC-A (Figure 3(a)). The corresponding kinetic reflectivity curve is also shown in Figure 3(b). The angular SPR reflectivity curve after immobilization of Anti-A was shifted to higher angle, indicating that Anti-A was immobilized on the sensor chip. The increase in the reflectivity during the immobilization is also shown in the SPR kinetic curve (Figure 3(b)). However, the reflectivity during the detection of RBC-A is almost constant, as shown in the kinetic curve (Figure 3(b)). Moreover, the corresponding angular reflectivity curve was almost constant after the detection of RBC-A. This indicates that the conventional SPR sensor is limited to the detection of RBC, presumably because of the fact that the evanescence field of conventional SPR is much shorter than the thickness of RBCs, and hence the reflectivity change is not sensitive to the adsorption of the surface.

Then, the LR-SPR sensor chip was used for the detection and classification of RBC typing on 11-MUA SAM. The LRSPR kinetic reflectivity curve for the immobilization of Anti$A$ and the detection of RBC-A is shown in Figure 4(b). The Anti-A was immobilized on activated group on the SAM surface. The remaining available active groups were blocked by ethanolamine. The baseline in the figure is obtained after the activation process by rinsing with PBS buffer. The reflectivity was obviously increased by the immobilization of Anti-A and after the detection of RBC-A (by 0.008) (Figure 4(b)). In this result, the baseline of the initial reflectivity is relatively high because the experiment was continuously carried out after the activation of the surface. Hence, the reflectivity was saturated by the injection of Anti-A. However, after the detection of RBC-A, we clearly observed the increased reflectivity change compared to the conventional SPR. In order to clearly compare with the conventional SPR reflectivity curve, the $y$-scale was shown by finite difference of reflectivity, $\Delta R$. Furthermore, the angular SPR reflectivity curve clearly shows 


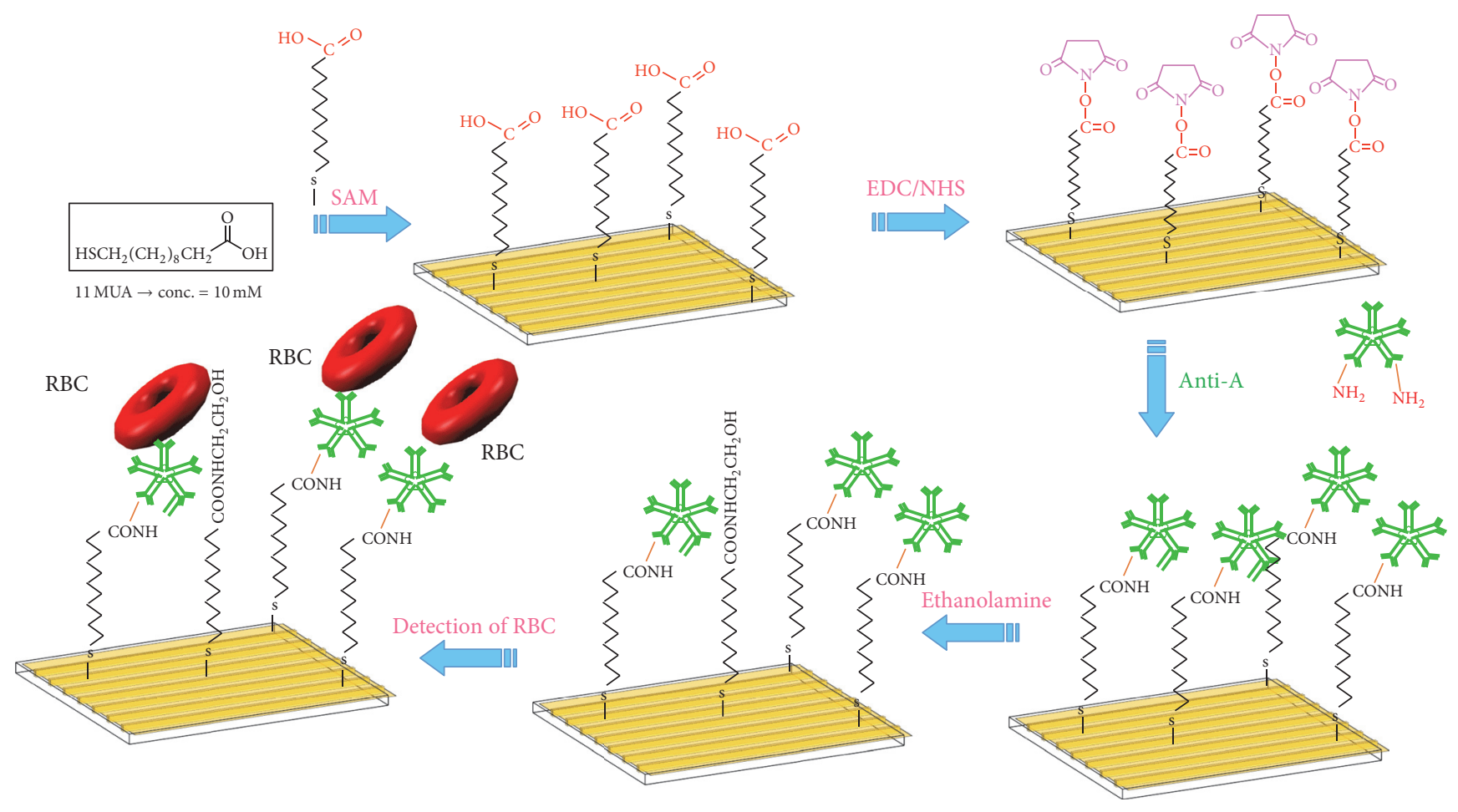

SAM = self-assembled monoplayer

$11 \mathrm{MUA}=11$-mercaptoundecanoic acid

$\mathrm{RBC}=$ red blood cell

FIGURE 2: Schematic representation of immobilization of antibodies (Anti-A/Anti-B) and detection of red blood cells (RBCs).

that the dip angle shifts to a higher angle after the detection of RBC-A (Figure 4(a)). These results indicate that the LR-SPR is more sensitive for the detection of RBC-A in comparison with the conventional short-range SPR measurement.

3.2. Surface Regeneration. Because one important advantage of the biosensor is reusability, we also studied the regeneration ability of RBC-A on Anti-A. For the regeneration of the RBC detection system, we found that $20 \mathrm{mM}$ of $\mathrm{NaOH}$ was a suitable condition to disrupt the antigen on the RBCs, causing the disruption of the RBC-antibody specific adsorption interaction without destroying immobilized antibody on the activated surface. The LR-SPR curve is shifted back to the lower incident angle and is almost the same as the LR-SPR curve of immobilized Anti-A surface, indicating that the adsorbed RBC-A is completely removed from the Anti-A surface without removing the immobilized Anti-A (Figure 4(a)). Figure 5 shows the kinetic property of the LR-SPR during the regeneration of RBC-A/Anti-A surface over three times. During the experiment, PBS buffer was used to obtain a baseline reading, which is shown as " $\Delta$ " in the figure. After the binding of RBC-A on the Anti-A for about $15 \mathrm{~min}$, the surface with residual unbound RBC-A was rinsed with PBS buffer, which is shown as " $\nabla$." Then, $20 \mathrm{mM}$ $\mathrm{NaOH}$ was injected on the RBC-A/Anti-A surface, followed by the injection of PBS buffer (baseline, shown as " $\Delta$ "). As shown in the figure, each time the baseline returned to the original baseline values, indicating that the Anti-A surface still remained (i.e., was regenerated), even after three sensing and regeneration processes.

3.3. Immobilization of Antibody and Detection of RBC-B. Next, we immobilized Anti-B on activated 11-MUA SAM surface and detected RBC-B by LR-SPR (Figure 6). In this experiment, the sensing procedure was the same as for RBCA detection described above. Similar to the results for RBC-A, LR-SPR showed an obvious increase at each adsorption step, indicating that it can easily detect RBC-B.

3.4. Specificity between Antibodies and RBCs. To study the specificity between antibodies and RBCs, Anti-A or Anti$B$ was immobilized on activated 11-MUA SAM surface, and RBC-A, RBC-B, or RBC-O was detected to determine both specific and nonspecific interactions. The LR-SPR kinetic curves during the detection of RBC-A, RBC-B, and RBC-O on Anti-A (Figure 7(a)) and Anti-B (Figure 7(b)) show that the reflectivity was increased in all cases. This indicated that the RBCs were adsorbed on the antibodies both by specific and nonspecific interactions. After rinsing with $\mathrm{PBS}$ buffer, the LR-SPR reflectivity for RBC-B and RBC-O decreased to around baseline. This is because the nonspecifically or physically adsorbed RBCs were removed from the Anti-A surface. On the other hand, the LR-SPR reflectivity for RBCA kept higher reflectivity than that of the initial baseline, 


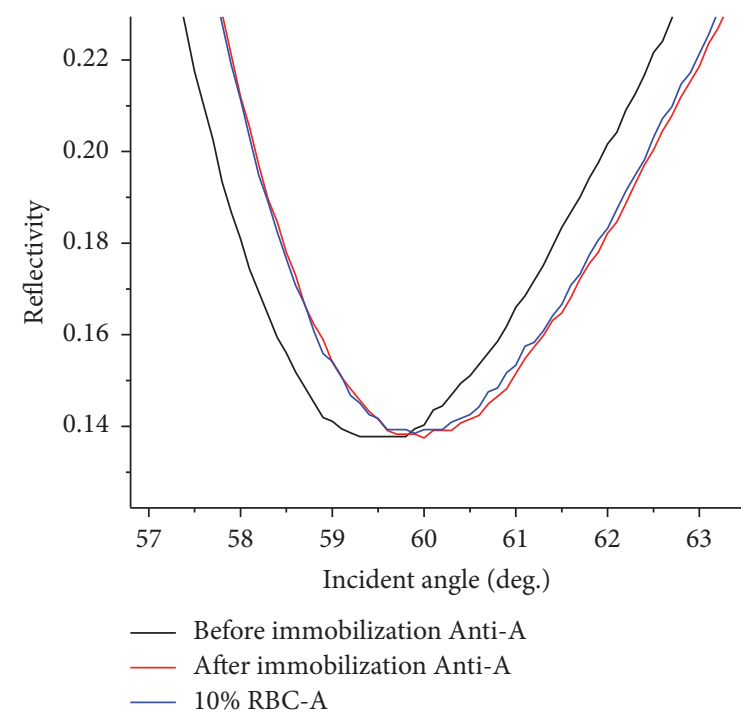

(a)

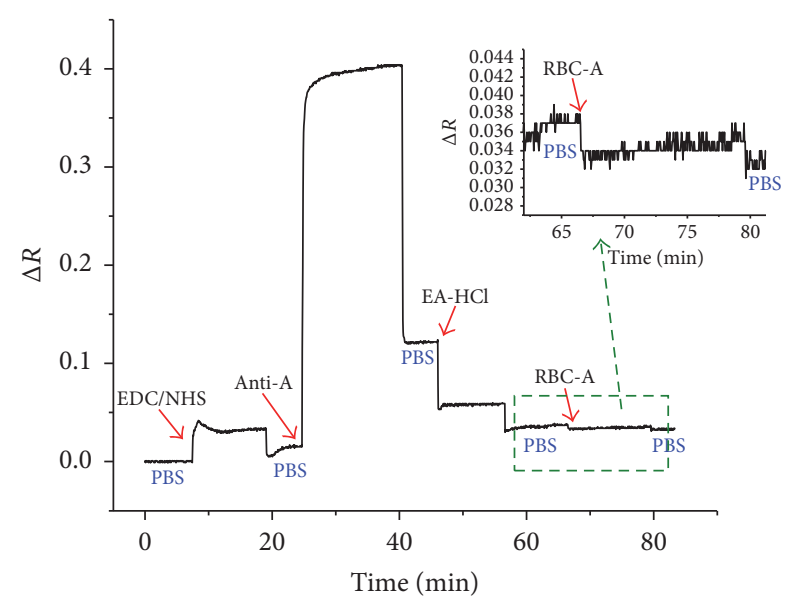

(b)

FIgURE 3: (a) Angular reflectivity curves of conventional SPR before and after immobilization and after the detection of RBC-A. (b) Corresponding SPR kinetic reflectivity curve.

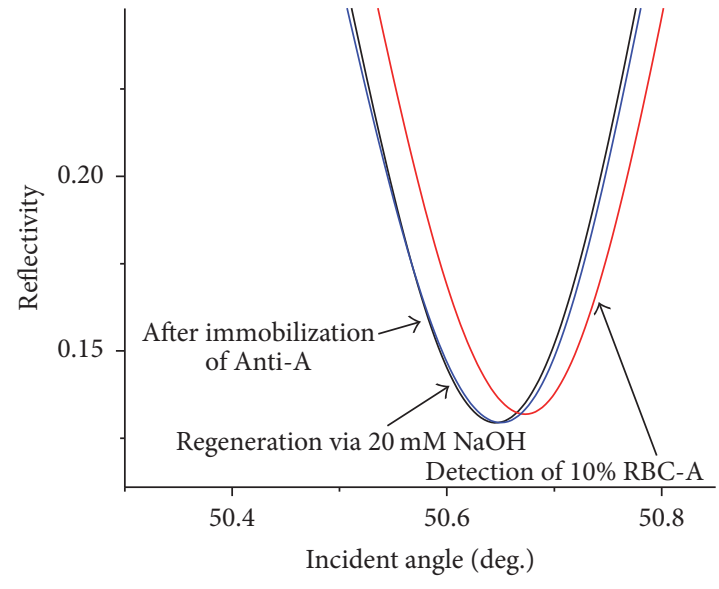

(a)

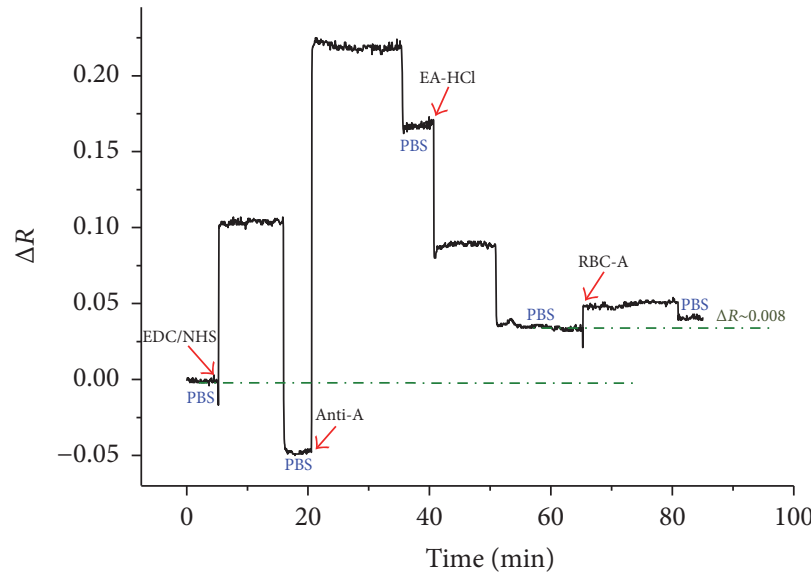

(b)

Figure 4: (a) Angular reflectivity curves of LR-SPR after immobilization of Anti-A, after detection of RBC-A, and after regeneration with $20 \mathrm{mM} \mathrm{NaOH}$. (b) Corresponding LR-SPR kinetic reflectivity curve.

indicating that the specifically adsorbed RBC-A on Anti-A remained on the surface. The slight decrease by the PBS buffer indicates some physically adsorbed RBC-A was removed from the surface. In the case of the RBCs detection on Anti$B$ surface, the LR-SPR reflectivity for RBC-A and RBC-O decreased to almost the initial baseline after the adsorption on the surface, while the reflectivity for RBC-B remained higher. These results indicate that the specific and nonspecific adsorption of RBCs can be clearly detected by the LR-SPR sensor chip, showing the ability to classify blood types.

3.5. Determination of the Detection Limit. Standard RBC-A and RBC-B samples were serially diluted from their original concentration and injected to study the interaction with
Anti-A and Anti-B immobilized on 11-MUA SAM. RBC-A and $\mathrm{RBC}-\mathrm{B}$ were detected on the immobilized Anti-A and Anti-B, respectively, by varying each concentration. RBCs at each concentration were detected for $10 \mathrm{~min}$, followed by rinsing with $\mathrm{PBS}$. The SPR reflectivity change $(\Delta R)$ was obtained by the reflectivity difference between the baseline before the injection of RBCs and after rinsing with the PBS buffer. Antibody surfaces were regenerated with $20 \mathrm{mM}$ $\mathrm{NaOH}$ for the following experiment. As shown in Figure 8, the reflectivity change $(\Delta R)$ of LR-SPR increases when the concentration of RBC-A and RBC-B increases. The limit of detection (LOD) of RBC-A and RBC-B were at $1.58 \times$ $10^{5}$ cells $/ \mathrm{ml}$ and $3.83 \times 10^{5}$ cells $/ \mathrm{ml}$, respectively. The LOD was defined as three times standard deviation of the blank (PBS buffer) [32]. We found that the obtained LOD using the 


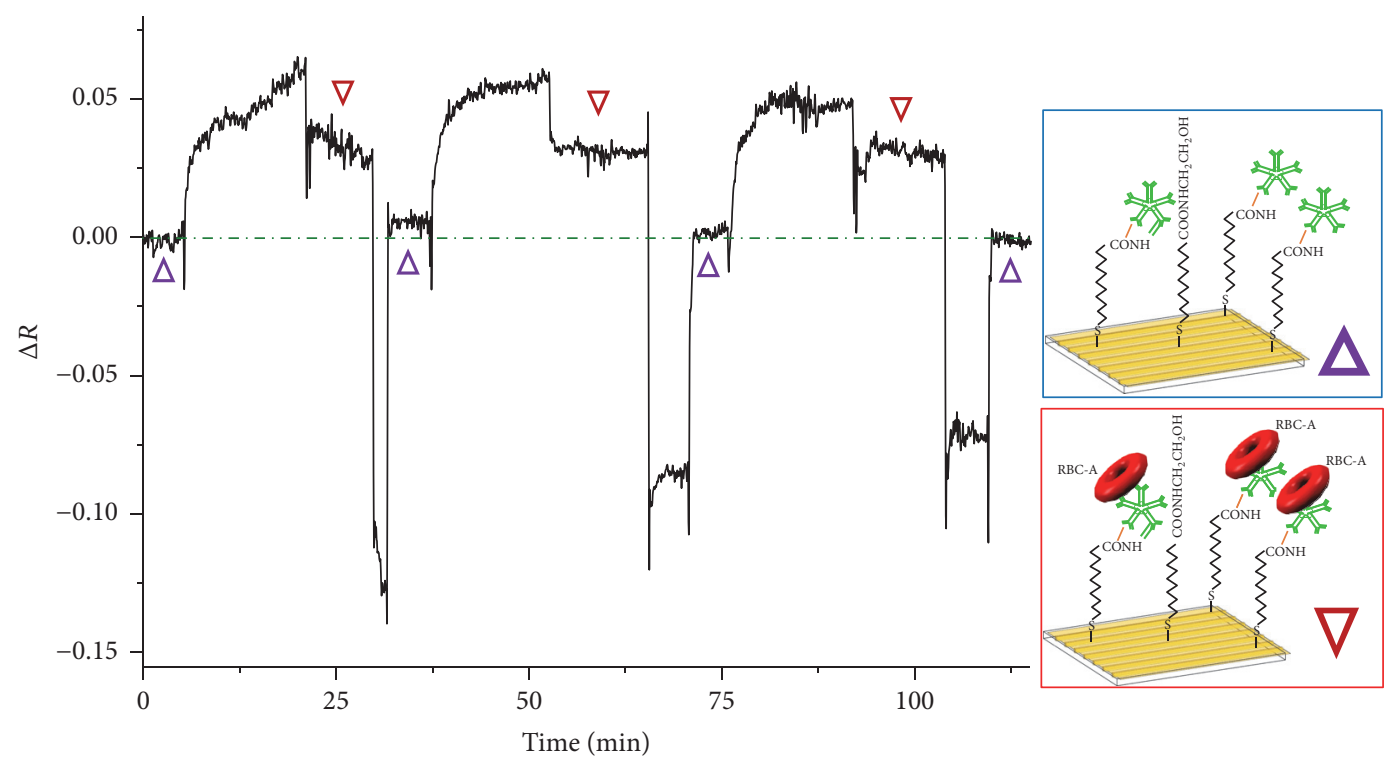

FIGURE 5: LR-SPR kinetic curve showing detection of red blood cells (RBCs) and regeneration of the surface using $20 \mathrm{mM}$ NaOH. PBS buffer was used to obtain a baseline reading $(\Delta)$ and then RBC-A is bound on the Anti-A for $\sim 5 \mathrm{~min}$, before the surface is rinsed with PBS buffer $(\nabla)$. Then, $20 \mathrm{mM} \mathrm{NaOH}$ is injected on the RBC-A/Anti-A surface, followed by the injection of PBS buffer $(\Delta)$.

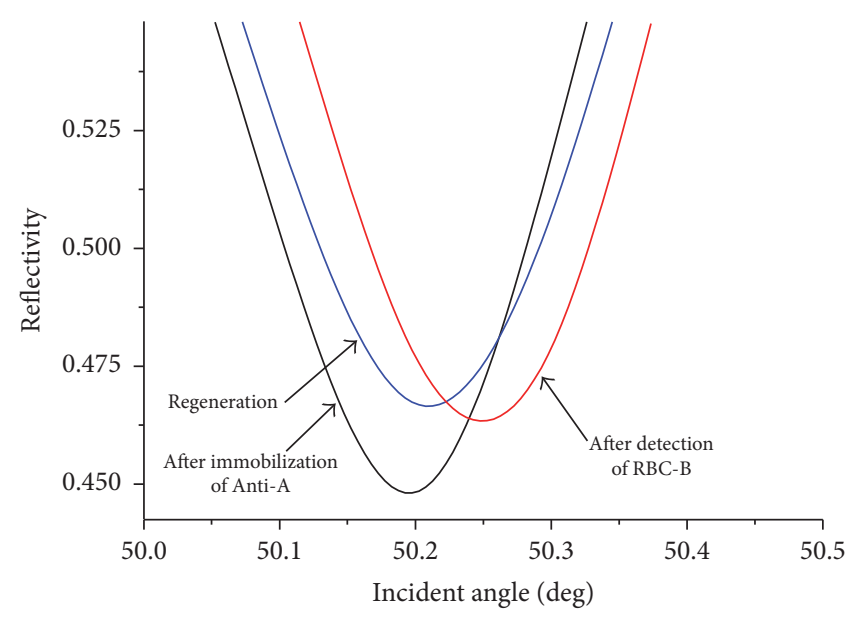

(a)

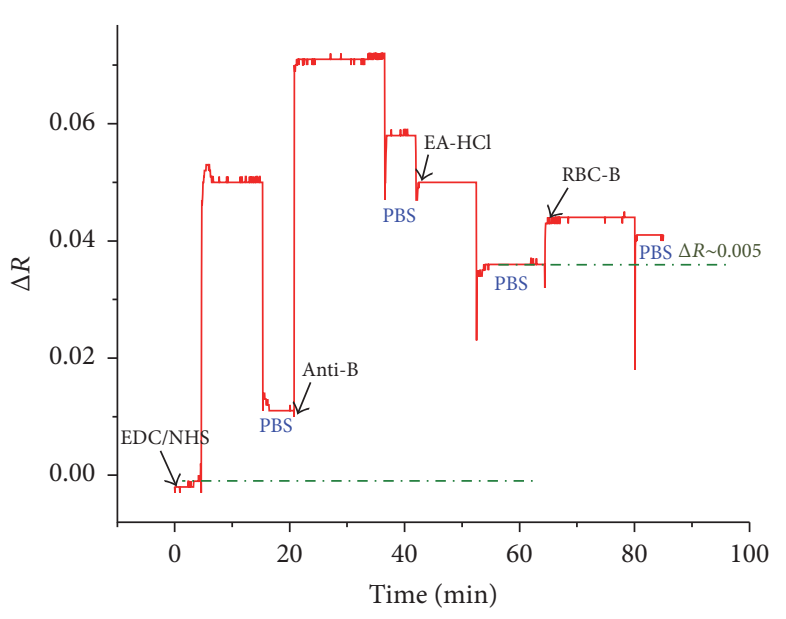

(b)

FIGURE 6: Angular reflectivity curves for LR-SPR after immobilization of Anti-B, after detection of RBC-B, and after regeneration with 20 mM $\mathrm{NaOH}$. (b) Corresponding LR-SPR kinetic reflectivity curve.

LR-SPR is lower than that with conventional short-range SPR (i.e., $3.3 \times 10^{8}$ cells $\left./ \mathrm{ml}\right)[25,26]$ and also lower than that with long-range surface plasmon waveguides that exhibited the LOD less than $3 \times 10^{5} \mathrm{cell} / \mathrm{ml}$ [33]. This clearly indicates that LR-SPR is a promising technique for the detection of RBCs.

\section{Conclusion}

We demonstrated that the LR-SPR sensor, consisting of highrefractive index glass, Cytop film layer, and thin gold ( $\mathrm{Au})$ film, is capable of detecting large analytes, red blood cell (RBCs). The antibodies of blood group A and group B (Anti$A$ and Anti-B) are able to be covalently immobilized on a grafting self-assembled monolayer (SAM)/Au surface on the
LR-SPR biosensor chip. For blood typing, the RBC samples are detected on the immobilized Anti-A and Anti-B surface by the change in the refractive index. We found that the results of blood typing obtained by the LR-SPR biosensor were consistent with those obtained from the agglutination test. Moreover, the LR-SPR exhibited the lowest detection limits of $1.58 \times 10^{5}$ cells $/ \mathrm{ml}$ for RBC-A and $3.83 \times 10^{5}$ cells $/ \mathrm{ml}$ for RBC-B, indicating that the LR-SPR chip has a higher sensitivity than conventional short-range SPR biosensors (3.3 $\times 10^{8}$ cells $/ \mathrm{ml}$ ). Finally, the sensor showed a good efficiency of surface regeneration using $20 \mathrm{mM} \mathrm{NaOH}$. Therefore, the LR-SPR technique demonstrates many advantages for the detection of RBCs and could be used to perform $\mathrm{ABO}$ blood group typing in the future. 


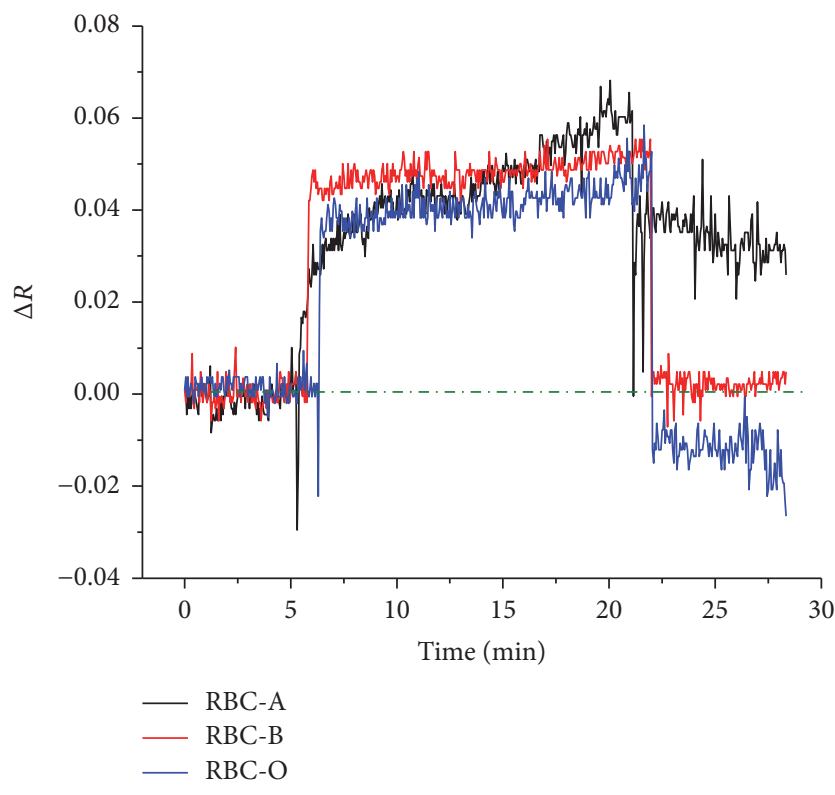

(a)

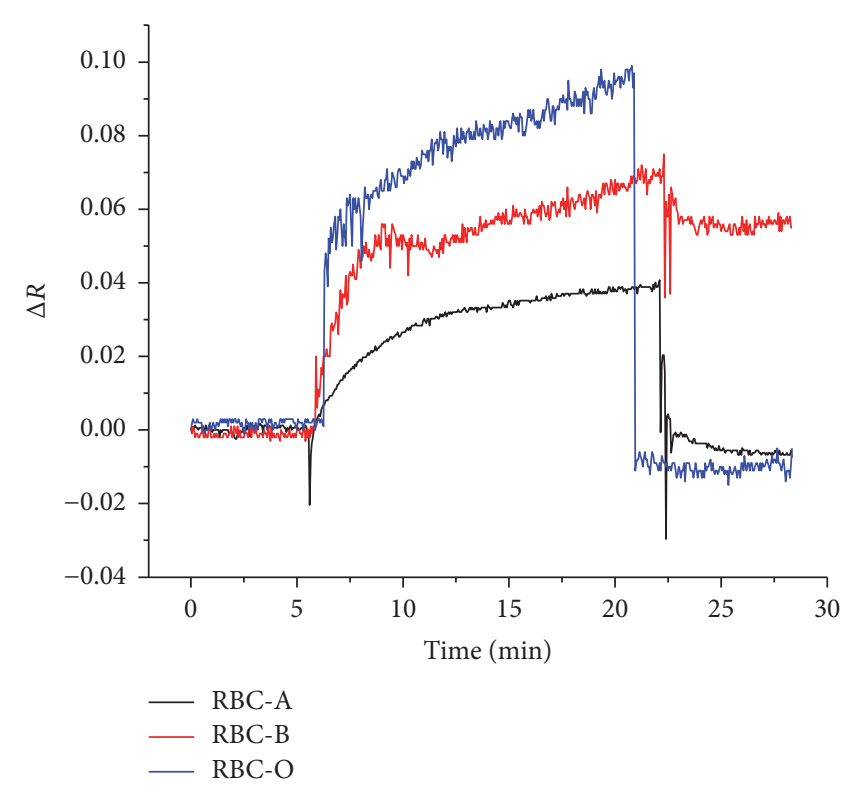

(b)

FIGURE 7: LR-SPR kinetic curves during the detection of RBC-A, RBC-B, and RBC-O on Anti-A (a) and on Anti-B (b).

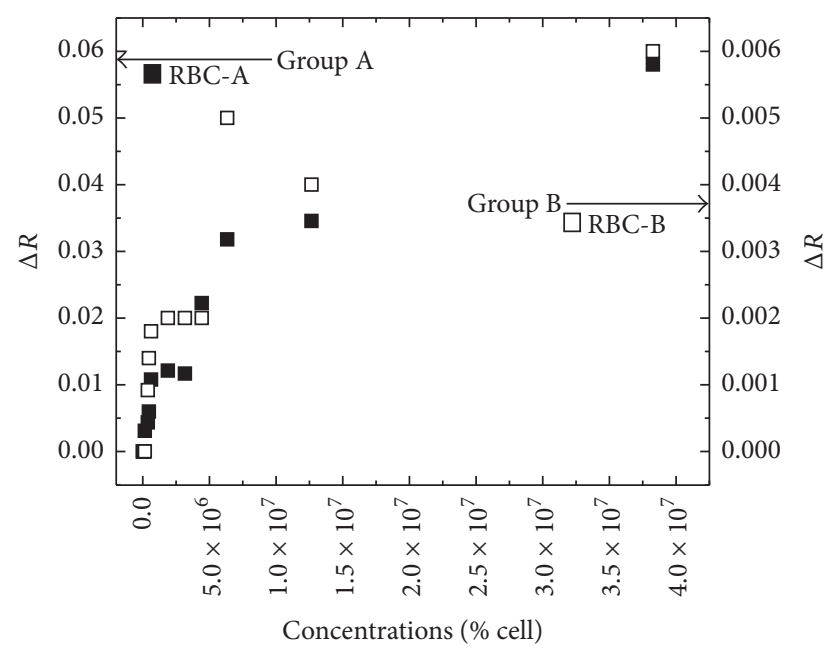

RBC-A

RBC-B

FIGURE 8: LR-SPR reflectivity change after sensing with various concentrations of red blood cells (RBCs).

\section{Competing Interests}

The authors declare that there is no conflict of interests regarding the publication of this paper.

\section{Acknowledgments}

This work was partly supported by a Grant-in-Aid for Scientific Research from the Japan Society for the Promotion of Science (JSPS) (16K13662).

\section{References}

[1] G. Daniels and I. Bromilow, Essential Guide to Blood Groups, Wiley-Blackwell, Malden, Mass, USA, 2nd edition, 2010.

[2] D. Thomas, "Blood transfusion in clinical medicine, 10th edition," Anaesthesia, vol. 54, no. 7, p. 722, 1999.

[3] G. Daniels, Human blood groups, Wiley-Blackwell, Malden, Massachusetts, USA, 3rd edition, 2013.

[4] J. D. Sweeney, Y. Rizk, and J. Sweeney, Clinical Transfusion Medicine, Landes Bioscience, 1999.

[5] W. Malomgré and B. Neumeister, "Recent and future trends in blood group typing," Analytical and Bioanalytical Chemistry, vol. 393, no. 5, pp. 1443-1451, 2009.

[6] J. Homola, "Present and future of surface plasmon resonance biosensors," Analytical and Bioanalytical Chemistry, vol. 377, no. 3, pp. 528-539, 2003.

[7] J. Homola, S. S. Yee, and G. Gauglitz, "Surface plasmon resonance sensors: review," Sensors and Actuators, B: Chemical, vol. 54, no. 1, pp. 3-15, 1999.

[8] G. J. Wegner, H. J. Lee, G. Marriott, and R. M. Corn, "Fabrication of histidine-tagged fusion protein arrays for surface plasmon resonance imaging studies of protein-protein and protein-DNA interactions," Analytical Chemistry, vol. 75, no. 18, pp. 4740-4746, 2003.

[9] R. Janmanee, A. Baba, S. Phanichphant et al., "In situ electrochemical-transmission surface plasmon resonance spectroscopy for poly(pyrrole-3-carboxylic acid) thin-film-based biosensor applications," ACS Applied Materials and Interfaces, vol. 4, no. 8, pp. 4270-4275, 2012.

[10] F.-C. Chien and S.-J. Chen, "A sensitivity comparison of optical biosensors based on four different surface plasmon resonance modes," Biosensors and Bioelectronics, vol. 20, no. 3, pp. 633642, 2004. 
[11] A. Baba, P. Taranekar, R. R. Ponnapati, W. Knoll, and R. C. Advincula, "Electrochemical surface plasmon resonance and waveguide-enhanced glucose biosensing with $\mathrm{N}$-alkylaminated polypyrrole/glucose oxidase multilayers," ACS Applied Materials and Interfaces, vol. 2, no. 8, pp. 2347-2354, 2010.

[12] S. Tian, A. Baba, J. Liu et al., "Electroactivity of polyaniline multilayer films in neutral solution and their electrocatalyzed oxidation of $\beta$-nicotinamide adenine dinucleotide," Advanced Functional Materials, vol. 13, no. 6, pp. 473-479, 2003.

[13] S. Löfås, M. Malmqvist, I. Rönnberg, E. Stenberg, B. Liedberg, and I. Lundström, "Bioanalysis with surface plasmon resonance," Sensors and Actuators: B. Chemical, vol. 5, no. 1-4, pp. 79-84, 1991.

[14] Surface Plasmons on Smooth and Rough Surfaces and on Gratings, vol. 111, Springer, Berlin, Germany, 1988.

[15] J. Dostálek, A. Kasry, and W. Knoll, "Long range surface plasmons for observation of biomolecular binding events at metallic surfaces," Plasmonics, vol. 2, no. 3, pp. 97-106, 2007.

[16] R. Slavík, J. Homola, and H. Vaisocherová, "Advanced biosensing using simultaneous excitation of short and long range surface plasmons," Measurement Science and Technology, vol. 17, no. 4, pp. 932-938, 2006.

[17] G. G. Nenninger, P. Tobiška, J. Homola, and S. S. Yee, "Longrange surface plasmons for high-resolution surface plasmon resonance sensors," Sensors and Actuators B: Chemical, vol. 74, no. 1-3, pp. 145-151, 2001.

[18] J. C. Quail, J. G. Rako, and H. J. Simon, "Long-range surfaceplasmon modes in silver and aluminum films," Optics Letters, vol. 8, no. 7, pp. 377-379, 1983.

[19] D. Sarid, "Long-range surface-plasma waves on very thin metal films," Physical Review Letters, vol. 47, no. 26, pp. 1927-1930, 1981.

[20] P. Netsuwan, H. Mimiya, A. Baba et al., "Long-range surface plasmon resonance immunosensor based on water-stable electrospun poly (acrylic acid) fibers," Sensors and Actuators B: Chemical, vol. 204, pp. 770-776, 2014.

[21] R. Slavík and J. Homola, "Ultrahigh resolution long range surface plasmon-based sensor," Sensors and Actuators, B: Chemical, vol. 123, no. 1, pp. 10-12, 2007.

[22] M. Vala, S. Etheridge, J. A. Roach, and J. Homola, "Long-range surface plasmons for sensitive detection of bacterial analytes," Sensors and Actuators, B: Chemical, vol. 139, no. 1, pp. 59-63, 2009.

[23] A. Kasry and W. Knoll, "Long range surface plasmon fluorescence spectroscopy," Applied Physics Letters, vol. 89, no. 10, Article ID 101106, 2006.

[24] C. J. Huang, J. Dostalek, and W. Knoll, "Optimization of layer structure supporting long range surface plasmons for surface plasmon-enhanced fluorescence spectroscopy biosensors," Journal of Vacuum Science and Technology B: Microelectronics and Nanometer Structures, vol. 28, no. 1, pp. 66-72, 2010.

[25] J. G. Quinn, R. O’Kennedy, M. Smyth, J. Moulds, and T. Frame, "Detection of blood group antigens utilising immobilised antibodies and surface plasmon resonance," Journal of Immunological Methods, vol. 206, no. 1-2, pp. 87-96, 1997.

[26] J. G. Quinn, S. O’Neill, A. Doyle et al., "Development and application of surface plasmon resonance-based biosensors for the detection of cell-ligand interactions," Analytical Biochemistry, vol. 281, no. 2, pp. 135-143, 2000.

[27] N. Houngkamhang, A. Vongsakulyanon, P. Peungthum et al., "ABO blood-typing using an antibody array technique based on surface plasmon resonance imaging," Sensors (Switzerland), vol. 13, no. 9, pp. 11913-11922, 2013.

[28] K. Sudprasert, P. Peungthum, A. Vongsakulyanon et al., "Evaluation of agglutination strength by a flow-induced cell movement assay based surface plasmon resonance (SPR) technique," Analyst, vol. 140, no. 3, pp. 880-888, 2015.

[29] O. Krupin, C. Wang, and P. Berini, "Selective capture of human red blood cells based on blood group using long-range surface plasmon waveguides," Biosensors and Bioelectronics, vol. 53, pp. 117-122, 2014.

[30] A. Baba, T. Mannen, Y. Ohdaira et al., "Detection of adrenaline on poly(3-aminobenzylamine) ultrathin film by electrochemical-surface plasmon resonance spectroscopy," Langmuir, vol. 26, no. 23, pp. 18476-18482, 2010.

[31] S. Sriwichai, A. Baba, S. Phanichphant, K. Shinbo, K. Kato, and F. Kaneko, "Electrochemically controlled surface plasmon resonance immunosensor for the detection of human immunoglobulin $\mathrm{G}$ on poly(3-aminobenzoic acid) ultrathin films," Sensors and Actuators, B: Chemical, vol. 147, no. 1, pp. 322-329, 2010.

[32] K. Nagatomo, T. Kawaguchi, N. Miura, K. Toko, and K. Matsumoto, "Development of a sensitive surface plasmon resonance immunosensor for detection of 2,4-dinitrotoluene with a novel oligo (ethylene glycol)-based sensor surface," Talanta, vol. 79, no. 4, pp. 1142-1148, 2009.

[33] A. W. Wark, H. J. Lee, and R. M. Corn, "Long-range surface plasmon resonance imaging for bioaffinity sensors," Analytical Chemistry, vol. 77, no. 13, pp. 3904-3907, 2005. 

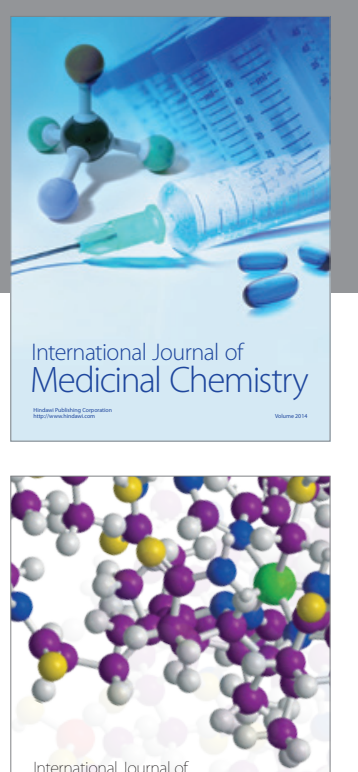

Carbohydrate Chemistry

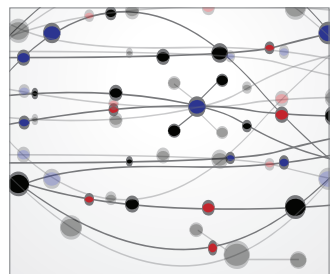

The Scientific World Journal
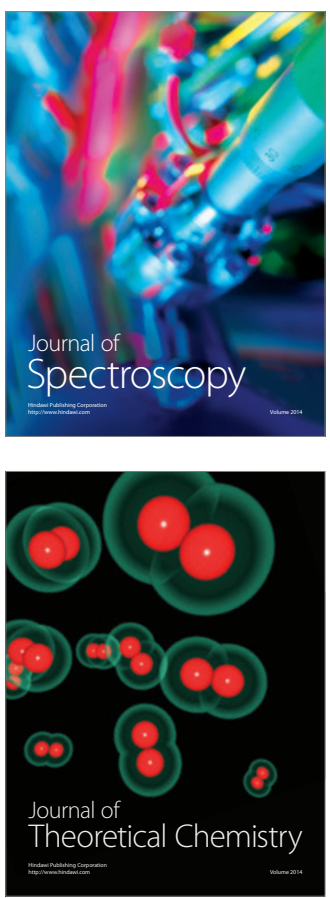
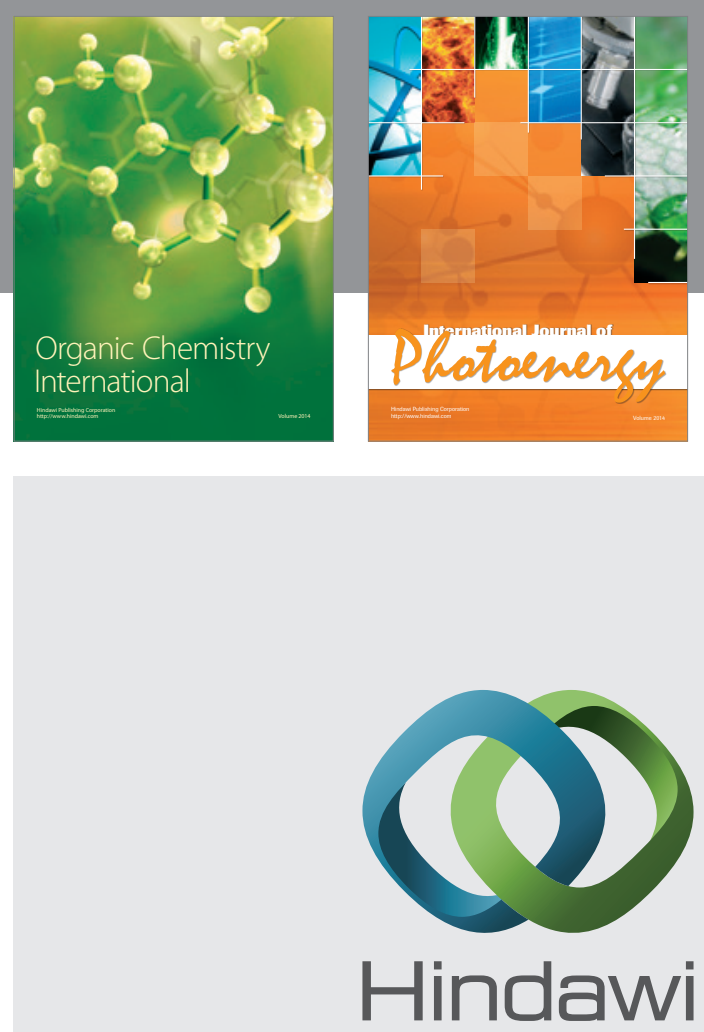

Submit your manuscripts at

http://www.hindawi.com

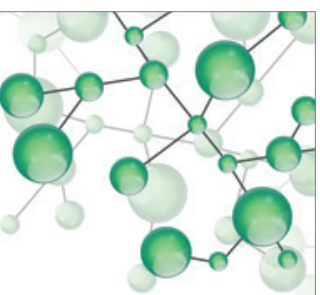

International Journal of

Inorganic Chemistry

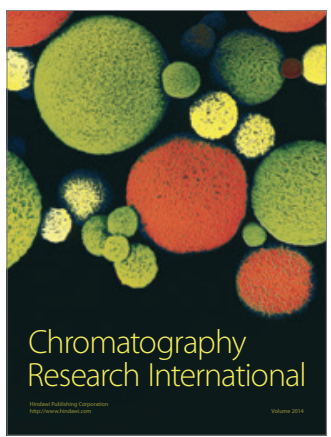

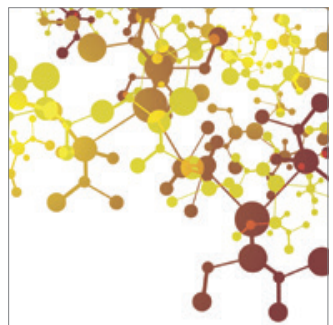

Applied Chemistry
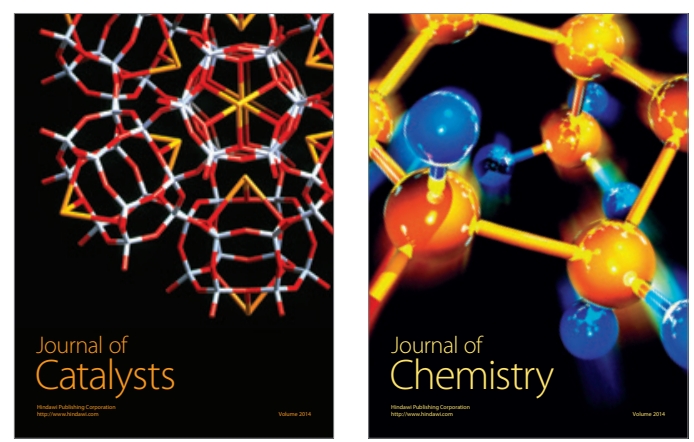
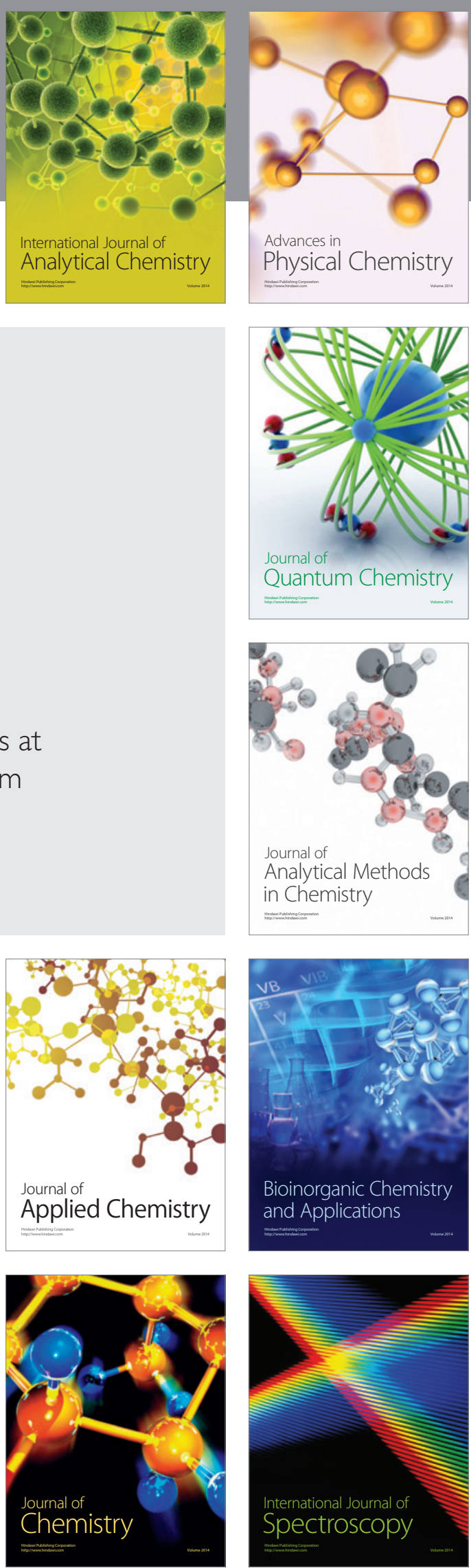